\title{
Magnetic distortion of the magnetotelluric tensor: a numerical study
}

\author{
A. K. Agarwal and J. T. Weaver \\ School of Earth and Ocean Sciences, University of Victoria, Victoria, BC, Canada, V8W $3 P 6$.
}

(Received March 15, 1999; Revised February 22, 2000; Accepted April 3, 2000)

\begin{abstract}
Decomposition of the magnetotelluric (MT) tensor when the electric field alone is subject to local galvanic distortion has received much attention in recent years. Recently some authors have extended such procedures to include the effects of the associated magnetic field distortion as well. With the aid of a three-dimensional modelling program the validity of the assumptions made in these analyses have been examined by considering the response over a range of periods of a small conductive cube at the surface of the earth and near a two-dimensional fault. The study indicates that the inclusion of magnetic distortion is necessary and important at short periods when induction occurs in the anomaly itself, but that the elements defining the magnetic distortion matrix become complex at such periods so that the assumptions underlying the theory are no longer valid. At longer periods the magnetic distortion matrix does become real and frequency-independent and therefore determinate, but its effect on the response becomes insignificant compared with that due to electric distortion. At these longer periods the phases of the regional impedances and the ratios of the electric distortion parameters are correctly recovered whether magnetic distortion is present or not. Calculations were repeated for a resistive cube, and also for a highly resistive region beneath the surface layer, with no significant enhancement of magnetic distortion. It is concluded that, at least for such models, inclusion of magnetic distortion in decompositions of the MT tensor does not, in general, offer any improvement over the conventional decompositions in which only distortion of the electric field is taken into account.
\end{abstract}

\section{Introduction}

For decomposition of the MT (or impedance) tensor, the assumption is often made that a measured 3D (three-dimensional) response is caused by frequency-independent galvanic distortion of a regionally $2 \mathrm{D}$ field. Such a phenomenon would occur if a small-scale, near-surface anomaly were embedded in a predominantly $2 \mathrm{D}$ geoelectric structure. The effect of the accumulation of electric charge on the boundary of the anomaly is to distort the local electric field and hence the $2 \mathrm{D}$ pattern of the induced current flow as well. All decomposition schemes take into account this local distortion of the electric field but most regard the anomalous magnetic field associated with the disturbed current flow as being too small to give rise to any significant distortion of the magnetic field.

Subsequently a more general decomposition scheme which included magnetic field distortion as well, was developed from first principles by Chave and Smith (1994) and applied to data from the Canadian shield and the EMSLAB experiment with the conclusion that magnetic distortion was present for periods below a few thousand seconds. The method was also applied by Chave and Jones (1997) to the BC87 dataset with the claim that the importance of including magnetic distortion effects was verified by improved interpretation of the data for periods greater than $10 \mathrm{~s}$ at some, but not all of the sites. White et al. (1997) have also assumed that magnetic as well as electric distortion is present

Copy right (c) The Society of Geomagnetism and Earth, Planetary and Space Sciences (SGEPSS); The Seismological Society of Japan; The Volcanological Society of Japan; The Geodetic Society of Japan; The Japanese Society for Planetary Sciences. in seafloor MT data in the Tasman Sea and on the Juan de Fuca plate. As far as we are aware, however, the validity of the theory, particularly the underlying assumptions about the nature of the electric and magnetic distortion matrices, has not yet been checked on 3D numerical models. Here we make a first attempt at this; only one synthetic model, consisting of a small anomalous cube at the surface of an earth traversed by a $2 \mathrm{D}$ vertical fault, has been considered, but we investigate the response and distortion effects for this single model over nearly five decades of periods ranging from 0.1 $\mathrm{s}$ to $5000 \mathrm{~s}$ and for an anomaly that is both conductive and resistive relative to its host medium.

\section{Electric and Magnetic Distortion}

The fundamental assumption underlying all decompositions of the MT tensor based on galvanic distortion is that the distorting anomaly is small enough and shallow enough that, over the frequency range of interest, the horizontal regional electric field is approximately uniform and equal to its surface value $\mathbf{E}^{r}$ over the full extent of the anomaly. This means that the charges that accumulate on the boundaries of the anomaly do so in phase with $\mathbf{E}^{r}$ and in a manner that is independent of frequency, and therefore that the horizontal galvanic electric field $\mathbf{E}^{g}$ associated with these charges is likewise in-phase with the regional field and frequencyindependent. Mathematically, this relationship is expressed as $\mathbf{E}^{g}=\mathbf{A} \cdot \mathbf{E}^{r}$ where $\mathbf{A}$ is a tensor represented by a $2 \times 2$ matrix whose elements are real and frequency-independent. Groom and Bahr (1992) and Chave and Smith (1994) have theoretically justified the validity of this assumption under the stated conditions by analysing the integral equation that 
governs the behaviour of the electric field. It follows that the measured (total) electric field

$$
\mathbf{E}^{m}=\mathbf{E}^{r}+\mathbf{E}^{g}
$$

can be written as

$$
\mathbf{E}^{m}=\mathbf{D} \cdot \mathbf{E}^{r}
$$

where $\mathbf{D}=\mathbf{I}+\mathbf{A}$ ( $\mathbf{I}$ is the unit tensor) is also real and frequency-independent. This is the familiar distortion equation used by Bahr (1988), Groom and Bailey (1989) and Smith (1997), when decomposing the MT tensor for regional structures that are 2D.

The measured magnetic field can be similarly separated into its regional and galvanic parts, the analagous equation to (1) for the horizontal magnetic field at the surface of the earth being

$$
\mathbf{B}^{m}=\mathbf{B}^{r}+\mathbf{B}^{g} .
$$

The galvanic component represents the magnetic field of the electric currents that are superimposed on the $2 \mathrm{D}$ regional current flow to give the distorted flow pattern around the small anomaly. In the work cited above the authors all postulated that $\mathbf{B}^{g}$ was negligible compared with the regional magnetic field, and therefore replaced (3) with $\mathbf{B}^{m}=\mathbf{B}^{r}$. More recent investigations by Chave and Smith (1994), Smith (1997) and Chave and Jones (1997), however, have accounted for the magnetic field associated with galvanic currents in the manner described below for which we follow the method and notation of Smith (1997).

Since $\mathbf{B}^{g}$ is the magnetic field of galvanic currents which are themselves in phase with the regional electric field $\mathbf{E}^{r}$, it follows that we may write

$$
\mathbf{B}^{g}=\mathbf{C} \cdot \mathbf{E}^{r}
$$

where $\mathbf{C}$ is a tensor whose elements are also real and frequency-independent. The $2 \times 2$ matrix representation of $\mathbf{C}$ is called the magnetic distortion matrix to distinguish it from the electric distortion matrix defined by D. Substituting (4) into (3), recalling that the measured MT tensor $\mathbf{Z}^{m}$ is defined by

$$
\mathbf{E}^{m}=\mathbf{Z}^{m} \cdot \mathbf{B}^{m}
$$

and making use of (2), we obtain

$$
\mathbf{D} \cdot \mathbf{E}^{r}=\mathbf{Z}^{m} \cdot\left(\mathbf{B}^{r}+\mathbf{C} \cdot \mathbf{E}^{r}\right) .
$$

The regional MT tensor $\mathbf{Z}^{r}$ relates $\mathbf{E}^{r}$ to $\mathbf{B}^{r}$ through an equation analogous to (5). Thus it is readily deduced from (6) that

$$
\mathbf{Z}^{m}=\mathbf{D} \cdot \mathbf{Z}^{r}-\mathbf{Z}^{m} \cdot\left(\mathbf{C} \cdot \mathbf{Z}^{r}\right) .
$$

If there is no magnetic distortion, i.e. if $\mathbf{C}=\mathbf{0}$, then (7) reduces to the conventional equation $\mathbf{Z}^{m}=\mathbf{D} \cdot \mathbf{Z}^{r}$ relating the measured and regional MT tensors when electric distortion alone is present.

\section{Computation of Tensor Elements}

We first make the assumption that all measurements are made in the principal frame of reference in which the axes are aligned parallel and perpendicular to the direction of strike in the regional 2D structure. How the axes can be rotated into this frame independently has been dicussed elsewhere, e.g. Smith (1997). For now we simply note that the diagonal elements of the regional MT tensor $\mathbf{Z}^{r}$ vanish in this frame, so that the tensor is defined by just two complex (four real) elements, $Z_{12}$ and $Z_{21}$.

Solving Eq. (7) is problematical because there are twelve real unknowns in the elements of the matrices of $\mathbf{C}, \mathbf{D}$ and $\mathbf{Z}^{r}$, but only eight real equations represented by the four complex elements of $\mathbf{Z}^{m}$, for any given frequency. In fact, Chave and Smith (1994) have shown that two of the unknowns, the off-diagonal elements of $\mathbf{C}$, are fundamentally indeterminate because they can assume arbitrary values, with correspondingly different values for $\mathbf{Z}^{r}$, in the decomposition expressed by Eq. (7), while another two, the amplitudes $g_{1}$ and $g_{2}$ in the representation

$$
\mathbf{D}=\left(\begin{array}{cc}
g_{1} \cos \phi_{1} & -g_{2} \sin \phi_{2} \\
g_{1} \sin \phi_{1} & g_{2} \cos \phi_{2}
\end{array}\right)
$$

are revealed as nothing more than undetermined scaling factors of the non-vanishing elements $Z_{12}^{r}$ and $Z_{21}^{r}$ in the regional MT tensor. Accordingly, Smith (1997) has suggested that the unknowns can be reduced to eight if $\mathbf{C}$ and $\mathbf{D}$ are defined as

$$
\mathbf{D}=\left(\begin{array}{ll}
1 & c \\
b & 1
\end{array}\right), \quad \mathbf{C}=\left(\begin{array}{ll}
\gamma & 0 \\
0 & \varepsilon
\end{array}\right)
$$

where $b=\tan \phi_{1}$ and $c=-\tan \phi_{2}$. This is equivalent to assigning the arbitrary values $g_{1}=\sec \phi_{1}$ and $g_{2}=\sec \phi_{2}$ in (8), and setting the off-diagonal elements of $\mathbf{C}$, which play no role in Eq. (7), to zero for simplicity. This latter assumption has the effect of adjusting the non-vanishing elements of the regional MT tensor to new values $\widetilde{Z_{12}}$ and $Z_{21}$ defined by

$$
\widetilde{Z_{12}^{r}}=Z_{12}^{r} /\left(1-C_{21} Z_{12}^{r}\right), \quad \widetilde{Z_{21}^{r}}=Z_{21}^{r} /\left(1-C_{12} Z_{12}^{r}\right)
$$

(Smith, 1997). The resulting (complex) components of (7) are

$$
\begin{aligned}
& Z_{11}^{m}=\varepsilon \operatorname{det} \mathbf{Z}^{m}+c Z_{21}^{m} \\
& Z_{12}^{m}=Z_{12}^{r}\left(1-\gamma Z_{11}^{m}\right) \\
& Z_{21}^{m}=Z_{21}^{r}\left(1-\varepsilon Z_{22}^{m}\right) \\
& Z_{22}^{m}=\gamma \operatorname{det} \mathbf{Z}^{m}+b Z_{12}^{m}
\end{aligned}
$$

Equation (11) has been simplified by substituting for $Z_{21}^{r}$ as given by (13), and similarly $Z_{12}^{r}$ has been eliminated from (14) with the aid of (12). The two real equations represented by the complex (11) can be solved to find $c$ and $\varepsilon$, and likewise (14) gives $b$ and $\gamma$. The regional tensor elements can then be recovered from (12) and (13).

\section{A Synthetic Model}

To test this theory and its underlying assumptions, a numerical model has been designed to represent galvanic distortion in a regional $2 \mathrm{D}$ setting. It comprises a small conductive cube located at the surface of the earth and near a $2 \mathrm{D}$ vertical fault, as shown in Fig. 1. Rectangular coordinates are defined with respect to an origin symmetrically placed on the surface relative to the cube and with the $x$-axis defined by the fault line, the $z$-axis directed vertically downwards into the earth, and the $y$-axis in the direction perpendicular to 


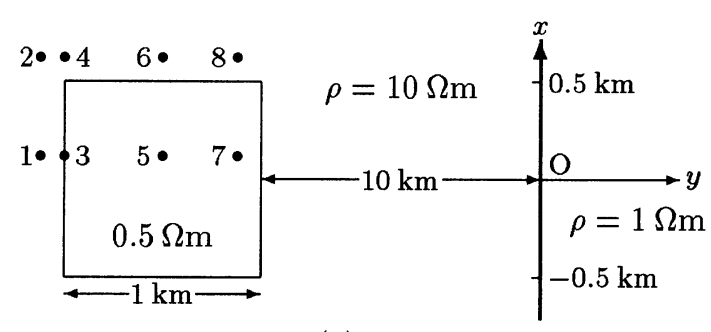

(a)

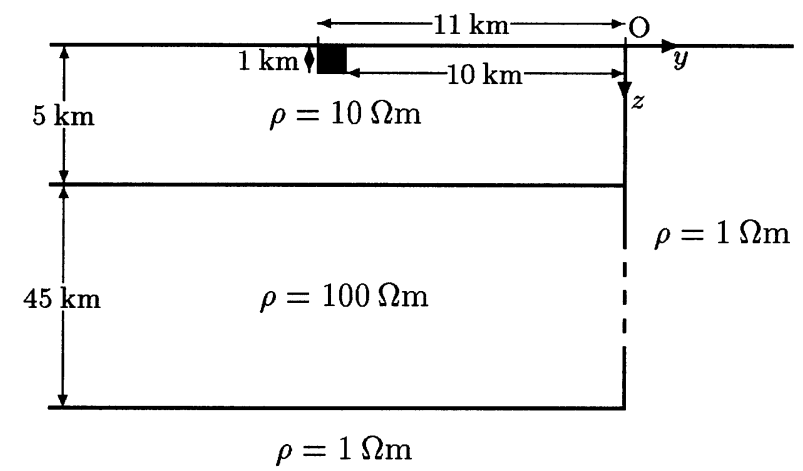

(b)

Fig. 1. (a) The surface plane $z=0$ viewed from above and (b) vertical cross-section in the plane $x=0$ of the model chosen for numerical investigation. The small anomaly is a $1 \mathrm{~km}^{3}$ cubical block of resistivity of $0.5 \Omega \mathrm{m}$ whose cross-section is represented by the black square in (b) and whose surface is the enlarged square in (a). Its centre is $10.5 \mathrm{~km}$ from a 2D fault dividing a two-layered slab $50 \mathrm{~km}$ thick from a conductive region of resistivity $1 \Omega \mathrm{m}$ which also underlies the slab. The positions of Sites $1-8$, at which the responses are calculated, are indicated by the black dots. Odd and even numbered sites lie, respectively, on the lines $x=0.12$ and $0.62 \mathrm{~km}$ at the points, in increasing order, $y=-11.12,-11.0,-10.5$ and $-10.12 \mathrm{~km}$.

the fault such that the axes are right-handed. By calculating the total electric and magnetic fields with the aid of a 3D modelling program (Weaver et al., 1999) and using definition (5), the 'measured' MT tensor $\mathbf{Z}^{m}$ was first obtained for the periods $0.1,1,10,50,100,500,1000$ and $5000 \mathrm{~s}$ and for the two polarizations of the regional magnetic field in the direction of (i) the positive $x$-axis parallel to the fault line (B-polarization) and (ii) the negative $y$-axis perpendicular to the fault line (E-polarization). The corresponding regional fields $\mathbf{E}^{r}$ and $\mathbf{B}^{r}$ were also computed using a 2D modelling program for the model in Fig. 1 with the anomalous conductive cube removed, and from them the 'true' regional MT tensor $\mathbf{Z}^{r}$ was likewise found. The horizontal magnetic field components, $B_{x}$ and $B_{y}$ are plotted against period in Figs. 2 and 3 for the eight selected sites near the anomaly indicated in Fig. 1. The magnitudes of the components have been normalized by the amplitude of the regional magnetic field at infinity (so that the total field tends to $B_{x}=1, B_{y}=0$ as $x \rightarrow \infty, y \rightarrow \infty$ in the B-polarization mode with respect to the 2D fault, and $B_{x}=0, B_{y}=-1$ in the E-polarization mode). For comparison, the undistorted $2 \mathrm{D}$ fields that are present in the absence of the small conductive anomaly are also shown. These results were then substituted in the complex vector Eq. (2) for each polarization of the regional field to yield eight real scalar equations from which the four complex elements of the electric distortion matrix $\mathbf{D}$ could be
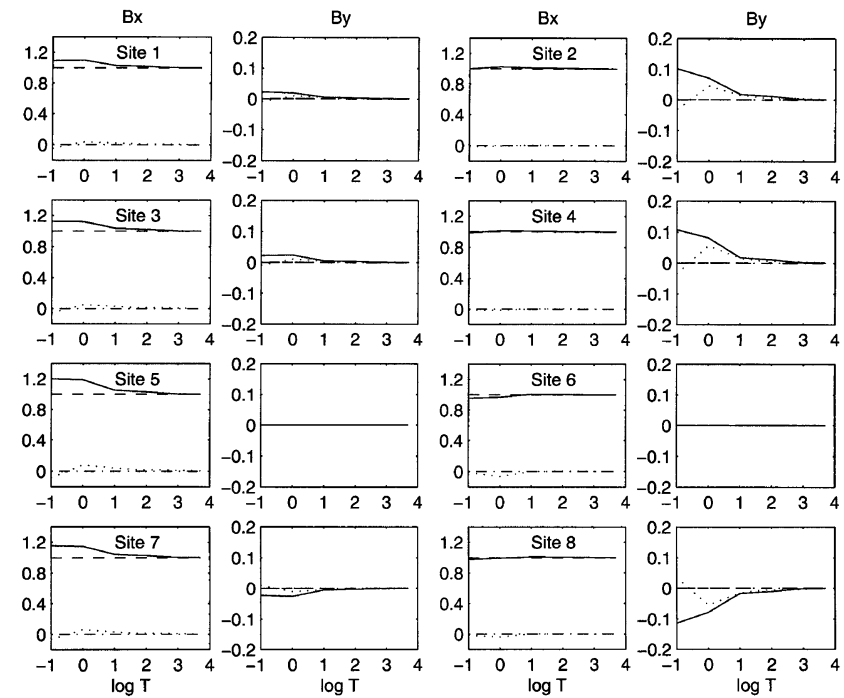

Fig. 2. Variation of the horizontal magnetic field components with period $T$ at Sites 1-8 shown in Fig. 1 when the regional magnetic field is in the $x$-direction (B-polarization). Solid and dotted lines represent, respectively, the variations of the real and imaginary parts of the magnetic fields. For comparison, the real (broken line) and imaginary (dot-dash line) parts of the regional field components, obtained when the anomalous cube in the model shown in Fig. 1 is removed, are also shown.
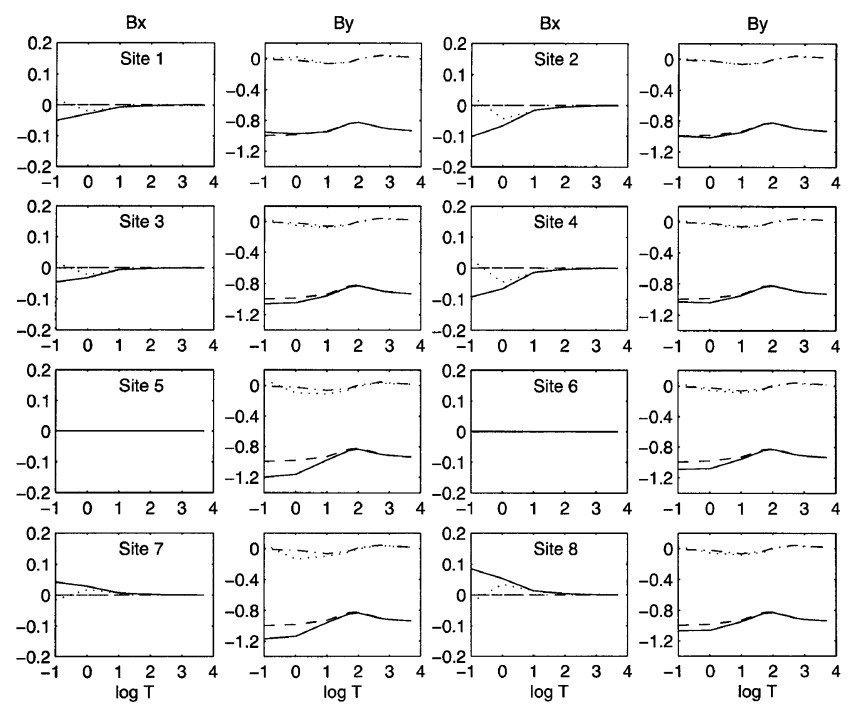

Fig. 3. As in Fig. 2 with the regional magnetic field in the direction of $-y$ (E-polarization).

calculated. (The elements are complex, in general, because no prior assumption is being made here about the nature of the distortion matrix - indeed it is the purpose of this exercise to test the validity of the assertion that the distortion matrix is, in fact, purely real.) Similarly, the complex equation

$$
\mathbf{C} \cdot \mathbf{E}^{r}=\mathbf{B}^{m}-\mathbf{B}^{r}
$$

which follows from (3) and (4), was used to obtain the complex elements of the magnetic distortion matrix $\mathbf{C}$. The elements of $\mathbf{D}$ and $\mathbf{C}$ are plotted as colour contour diagrams covering the surface of the model in Figs. 4 and 5 respectively, for the four representative periods 1, 10, 100 and 1000 $\mathrm{s}$ taken from the range of periods listed above. The broad range spanned by these four selected periods gives some sem- 


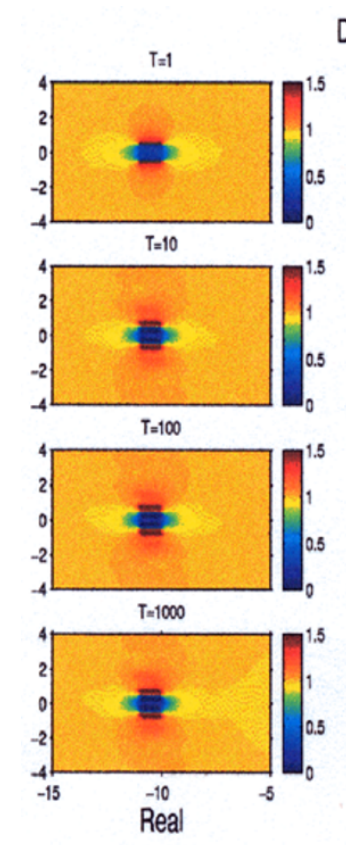

(a)
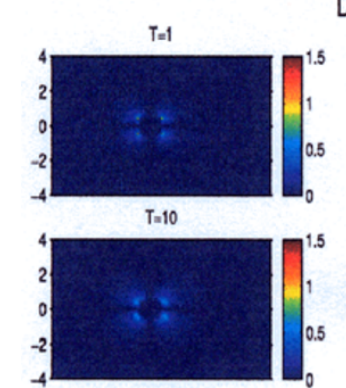

$T=100$

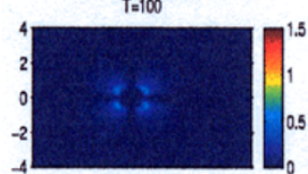

$T=1000$

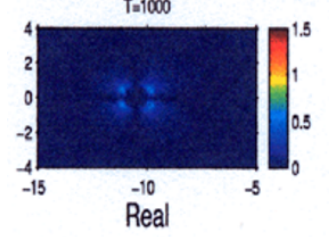

D21

011

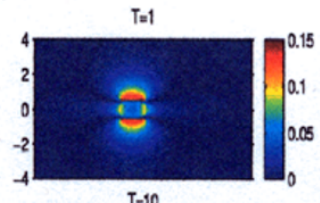

$T=10$

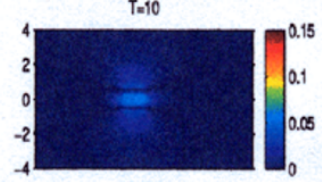

$T=100$

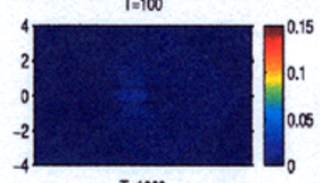

$T=1000$
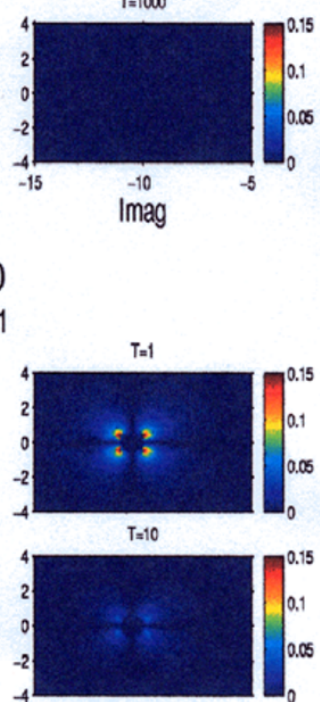

$T=100$

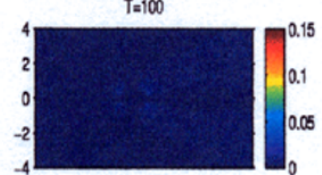

$T=1000$

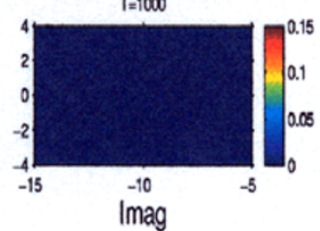

(c)
D12

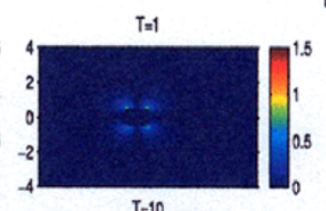

$T=10$

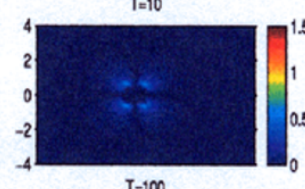

$T=100$

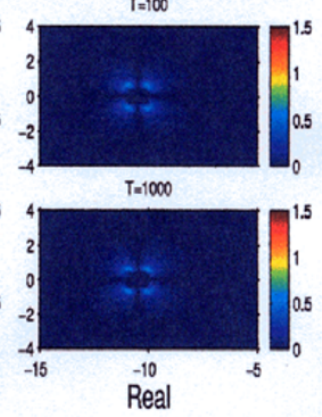

(b)
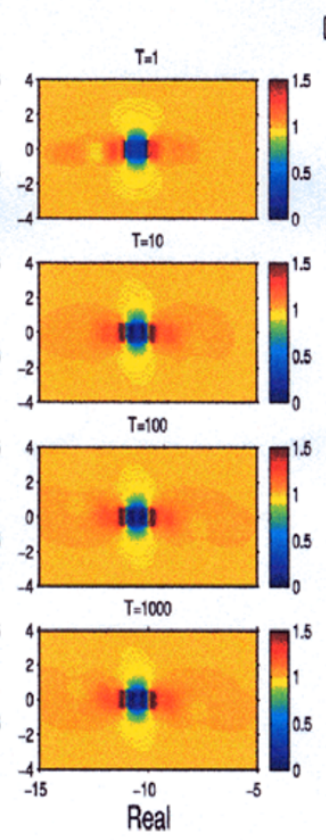

D22
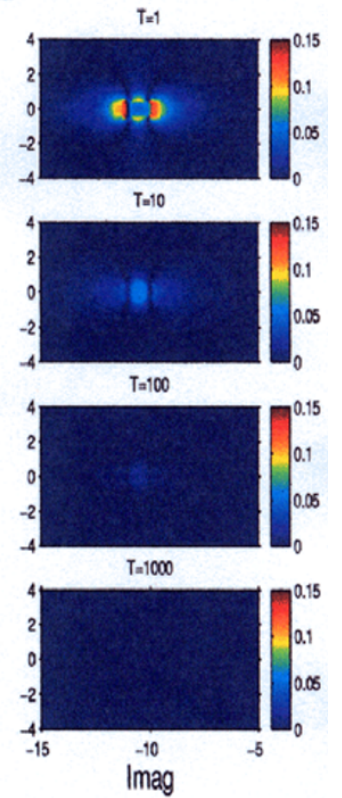

(d)

Fig. 4. Contour maps showing the variation across the surface plane $z=0$ of the (complex) elements (a) $D_{11}$, (b) $D_{12}$, (c) $D_{21}$ and (d) $D_{22}$, of the electric distortion matrix $\mathbf{D}$ for periods 1, 10, 100 and $1000 \mathrm{~s}$. The diagrams in the left-hand columns depict the values of the real parts of the elements, and those in the right-hand columns depict the imaginary parts.

blance of the period dependence of the real and imaginary parts of the elements of $\mathbf{D}$ and $\mathbf{C}$.

These 'true' results were used as benchmarks against which the validity of assuming that the distortion elements of $\mathbf{D}$ and $\mathbf{C}$ are real and frequency-independent could be tested, and from which the values of the distortion elements $b, c, \gamma, \varepsilon$, and the two regional impedances $\mu_{0} Z_{12}^{r}, \mu_{o} Z_{21}^{r}$ ( $\mu_{0}$ is vacuum permeability) as given by Smith's (1997) analysis described by Eqs. (11)-(14) above, could be compared. In the first two columns of Figs. 6 and 7, calculated values of $b$ and $c$ at the eight selected sites shown in Fig. 1 are compared with the real parts of the corresponding off-diagonal elements of the true electric distortion matrix $\mathbf{D}$ in which the columns have been normalised so that the diagonal elements are unity. In the second two columns, the values of $\gamma$ and $\epsilon$ at the same eight sites are compared with the real parts of the diagonal elements of the true magnetic distortion matrix C. Finally, Figs. 8 and 9 compare calculated and true regional impedances plotted in the form of apparent resistivity and phase given by $\mu_{0} T\left|Z_{i j}\right|^{2} / 2 \pi$ and $\arg Z_{i j}, \quad(i, j=1,2)$ 
C11

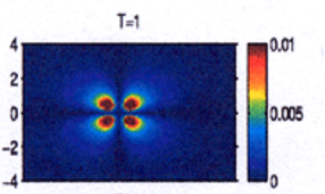

$T=10$

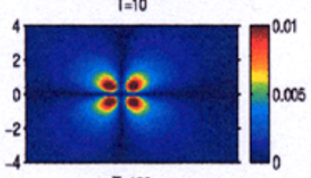

$T=100$

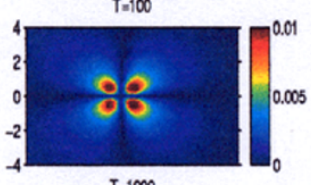

$T=1000$

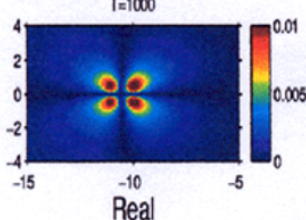

Real

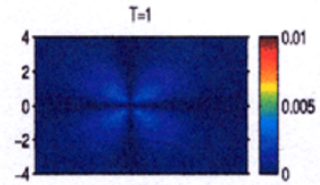

$T=10$

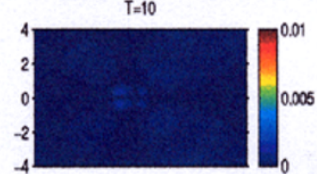

$T=100$

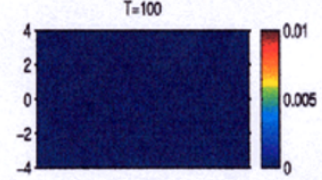

$T=1000$

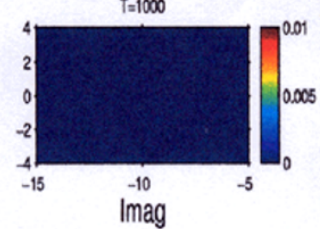

(a)

C21

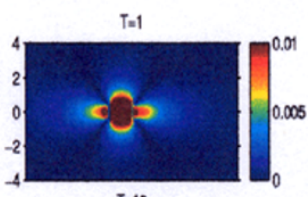

$T=10$

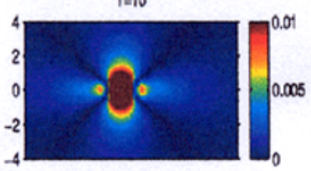

$T=100$

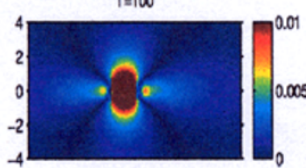

$T=1000$

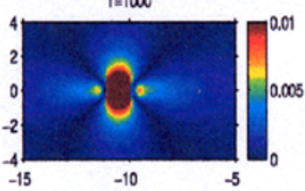

Real

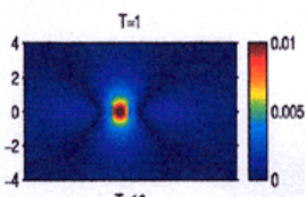

$T=10$

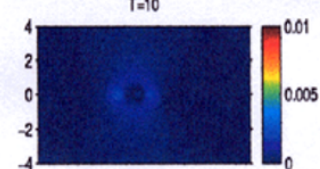

$T=100$

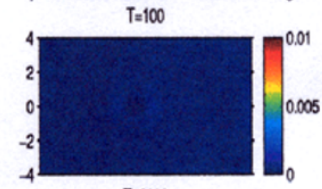

$T=1000$

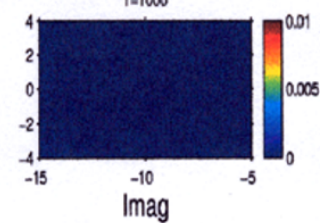

(c)
C12

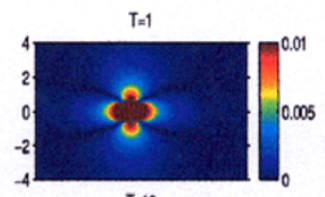

$T=10$

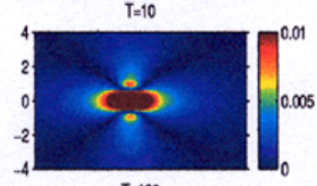

$T=100$
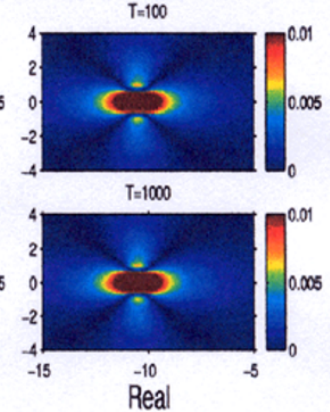

(b)

(22
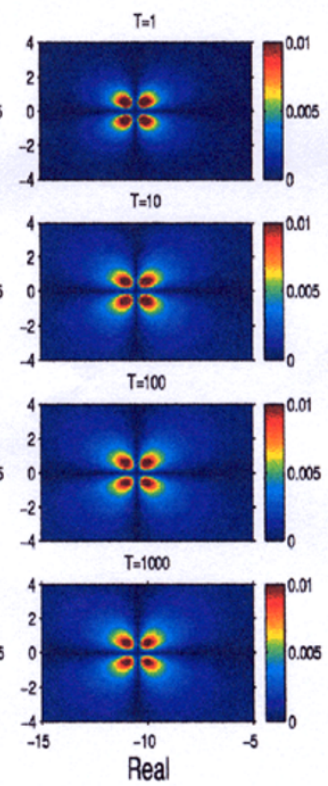

(d)
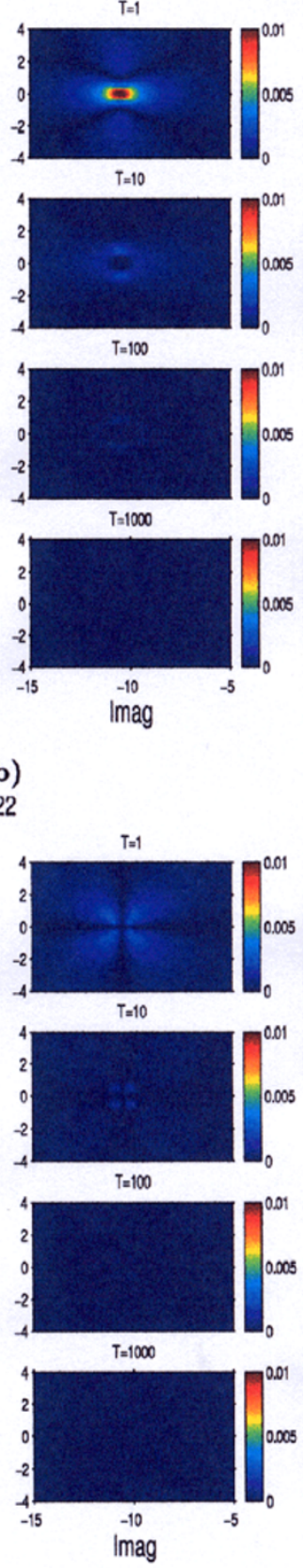

$T=10$

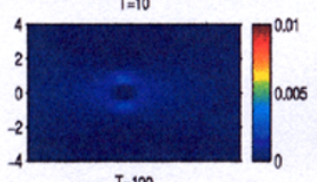

$T=100$
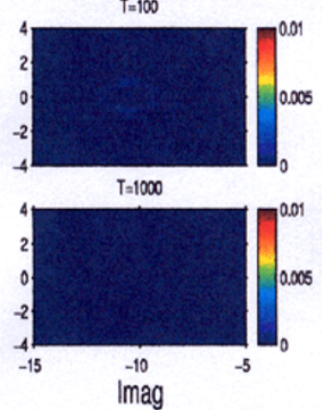

Fig. 5. Contour maps of the elements of the magnetic distortion matrix $\mathbf{C}$ corresponding to those in Fig. 4 for the electric distortion matrix. The values indicated by the scales on the right of the maps are in units of $\mathrm{s} / \mathrm{km}$.

respectively, where $T$ is the period of the field variations. Eqs. (11) and (13) of Smith's analysis yield values of $Z_{12}$ and $Z_{21}$ that have been scaled by factors $g_{1}$ and $g_{2}$ which make the diagonal elements of (8) unity, and they are also adjusted according to the formulae (10) as a result of the assumption that the off-diagonal elements of $\mathbf{C}$ vanish. In this synthetic example, the unknown scaling factors have been estimated from the real parts of the true (unnormalised) diagonal elements of $\mathbf{D}$, and likewise the real parts of the off-diagonal elements of $\mathbf{C}$ have been used to make the adjustments described in (10). Thus the calculated values of the regional apparent resistivities in Figs. 8 and 9 have been adjusted by using estimates obtained from the true values of $\mathbf{D}$ and $\mathbf{C}$, and the phases have also been modified using the true values of $\mathbf{C}$. With real data, it would be impossible to make such corrections, of course, unless the scaling factors and off-diagonal elements of $\mathbf{C}$ could be estimated independently.

\section{Discussion of the Numerical Results}

It is apparent from the diagrams in Figs. 4 and 5 that the fundamental assumption that the elements of the electric and magnetic distortion matrices are real and frequencyindependent is approximately valid for periods above the transitional range 10-100 s when the skin depth inside the 


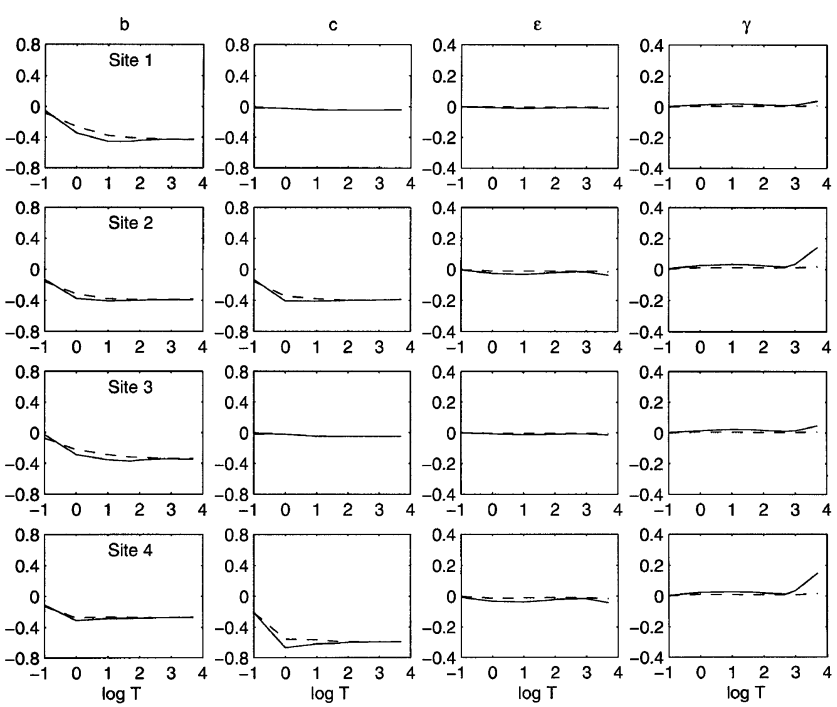

Fig. 6. Variation of the real electric distortion parameters, $b$ and $c$, (first and second columns respectively) and the real magnetic distortion parameters, $\varepsilon$ and $\gamma$ (third and fourth columns respectively, and plotted in units of $\mathrm{s} / \mathrm{km}$ ), with period $T$ at the Sites 1-4 shown in Fig. 1. The solid lines depict the values obtained using the analysis of Smith (1997)—Eqs. (11)-(14) in this paper; the broken lines are the corresponding real parts of the diagonal elements of the true values of $\mathbf{C}$ and $\mathbf{D}$ whose contour maps are shown in Figs. 4 and 5.

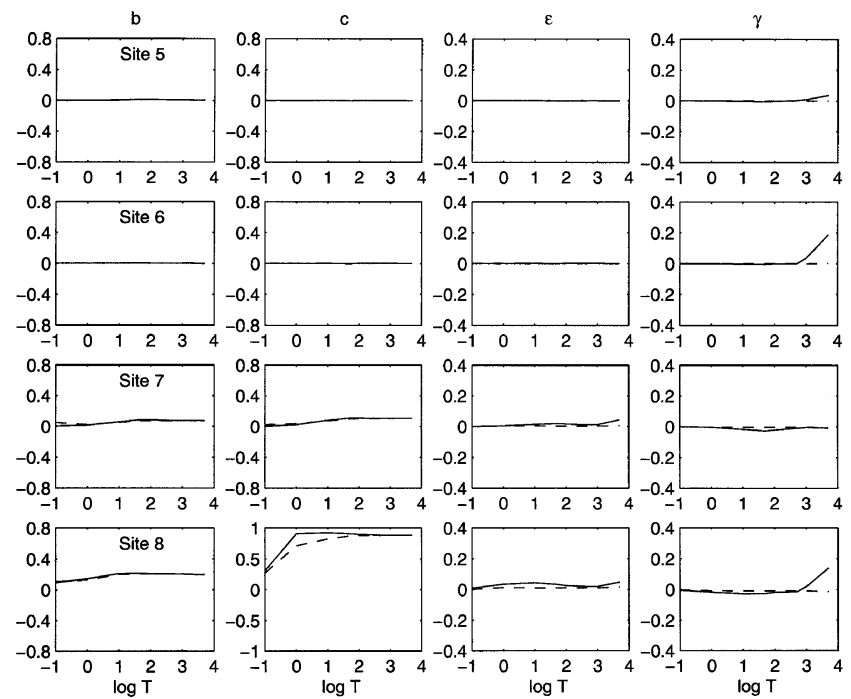

Fig. 7. As in Fig. 6 but for Sites 5-8.

anomalous cube starts to become significantly greater than the cube's dimensions. At shorter periods the cube can clearly no longer be considered small, 3D induction in the cube becomes increasingly important, and the elements of $\mathbf{D}$ and $\mathbf{C}$ become complex and frequency-dependent. But the curves plotted in Figs. 2 and 3 reveal that the total (distorted) horizontal magnetic field does not differ appreciably from the regional field for periods above the transitional range 10-100 s. In other words, magnetic distortion is insignificant over the very range of periods for which the basic assumptions of real and frequency-independent distortion matrices hold. Where the total horizontal magnetic field is markedly different from the regional field in Figs. 2 and 3-for periods shorter than $10 \mathrm{~s}$ - the inclusion of magnetic distortion is clearly impor-
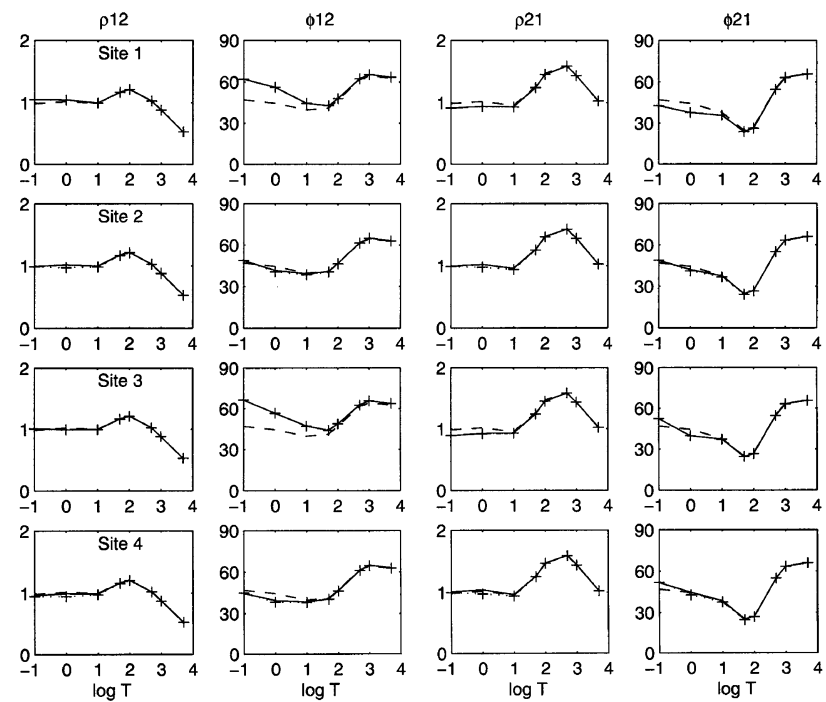

Fig. 8. Variation with period $T$ of the regional apparent resistivities $\rho_{12}$ and $\rho_{21}$, and the associated phases $\phi_{12}$ and $\phi_{21}$, at Sites 1-4 shown in Fig. 1. The values calculated from Eqs. (12) and (13) corrected with the aid of estimated scaling factors from $\mathbf{D}$ and off-diagonal elements of $\mathbf{C}$ (solid lines), are compared with the true values obtained by direct calculation (broken lines). The crosses indicate the values obtained on the assumption that only electric distortion is present $(\mathbf{C}=0)$.
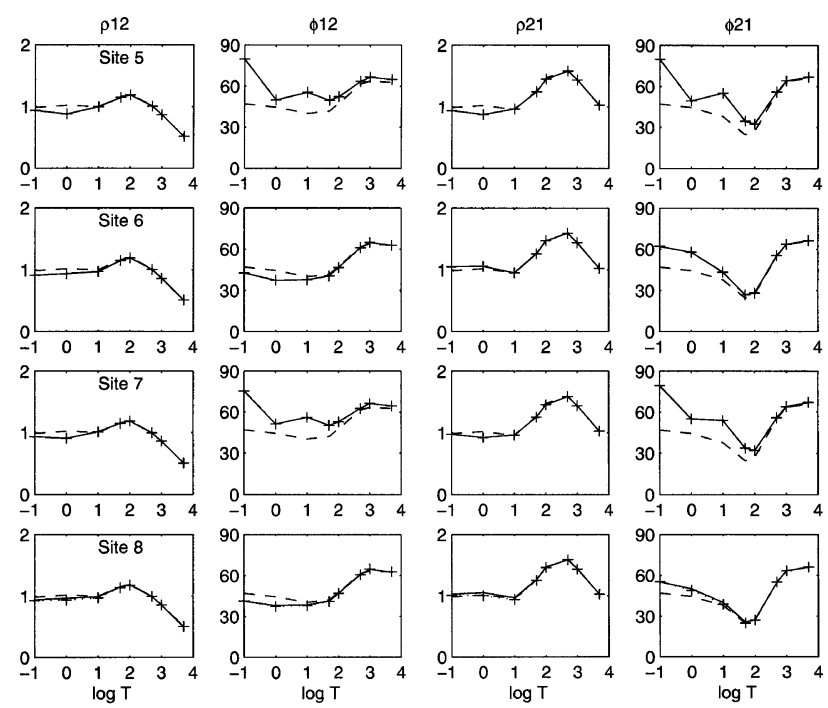

Fig. 9. As in Fig. 8 for Sites 5-8.

tant and necessary, but the distortion matrices are decidedly complex in this range so that the assumptions on which the theory of the tensor decomposition is based no longer hold.

Although magnetic distortion appears to be small for periods above the 10-100 s range, the analysis of Section 3 can still be applied in principle. In Figs. 6 and 7 it can be seen that while the electric distortion parameters $b$ and $c$ are accurately recovered at all the sites by Smith's analysis for periods above about $50 \mathrm{~s}$, the magnetic distortion parameters $\gamma$ and $\varepsilon$ are poorly resolved, in general, at long as well as short periods. In Figs. 8 and 9 the regional apparent resistivities are shown to be very well recovered for periods above $10 \mathrm{~s}$ while the corresponding phases are correctly reproduced for periods above $100 \mathrm{~s}$. The accuracy of the apparent resistiv- 
ities is somewhat misleading, of course, because the scaling factors $g_{1}$ and $g_{2}$ in the electric distortion matrix $\mathbf{D}$, which are normally unknown, have been estimated in this synthetic example from true values that are unavailable with real data. An interesting observation is that the calculated values of apparent resistivity and phase in Figs. 8 and 9 are virtually unaffected whether or not the magnetic distortion matrix $\mathbf{C}$ is included in the analysis. This implies that the corrections specified by formulae (10) are insignificant; only the scaling factors in $\mathbf{D}$ are important and they affect only the calculated apparent resistivities.

The calculations have been repeated for various other models in which the block is resistive rather than conductive relative to its host medium, and in which the top layer is highly insulated from the deep structure so that it can support a large regional current flow. In the first model, we simply changed the resistivity of the anomalous block to $200 \Omega \mathrm{m}$; in the second the resistivity of the anomaly was kept at $0.5 \Omega \mathrm{m}$, but the resistivity of the region beneath the surface layer was raised to $10^{4} \Omega \mathrm{m}$; and in the third model we combined and extended these alterations by including a $200 \Omega \mathrm{m}$ anomaly in a host surface layer of resistivity $1 \Omega \mathrm{m}$ (in order to enhance the regional current flow even more), raising the resistivity of the right slab to $10 \Omega \mathrm{m}$ (in order to preserve the regional twodimensionality), and retaining the highly resistive underlying slab on the left. In each case the magnetic field responses were calculated at the Sites 1, 2, 7 and 8 for a period of 100 s. As expected, the effect in B-polarization of replacing the conductive block with a resistive one was to diminish the real part of the distorted magnetic field $B_{x}$ compared with the regional field, rather than enhancing it as in Fig. 2, and to reverse the sign of the (very small) anomalous field $B_{y}$ which is consistent with current deflection rather than current gathering. In E-polarization the roles of $B_{x}$ and $B_{y}$ and their relative behaviour for resistive and conductive blocks were interchanged. For the other two models, which supported stronger regional currents in the surface layer, the magnitude of the anomalous magnetic field did increase slightly in the vicinity of the block, and still exhibited a definite trend consistent with the physics of the model, but it was still less than $4 \%$ of the regional field. Consequently, magnetic distortion effects remained insignificant.

The results of our investigation suggest, at least for this particular model which is a typical representation of galvanic distortion of an otherwise $2 \mathrm{D}$ regional field, that magnetic distortion plays but a negligible role in the decomposition of the MT tensor. It becomes important only at short periods where, in any case, the fundamental assumption of real, frequency-independent distortion breaks down, while at longer periods where the assumption is valid but magnetic distortion is insignificant, the analysis that has been proposed fails to yield accurate values of the (small) magnetic distortion parameters. The phases of the regional (decomposed) MT elements are, however, very well recovered for the longer periods even when magnetic distortion is completely ignored. Although we have considered only the one idealized but representative model of small-scale and in-phase distortion, we have found no evidence to suggest that the introduction of magnetic distortion into the usual MT tensor decomposition schemes provides any significant advantage for a small 3D surface anomaly; accounting for electric distortion alone appears to be quite sufficient. Since the regional magnetic field is attenuated by the screening effect of the ocean, magnetic distortion effects on seafloor MT data, are likely to be much more significant than for the simple synthetic model investigated here. Our conclusions apply only to land-based measurements.

Acknowledgments. Constructive comments from Hisashi Utada and an anonymous reviewer were very much appreciated. Support in the form of research grants from the Natural Sciences and Engineering Research Council of Canada and the University of Victoria is gratefully acknowledged.

\section{References}

Bahr, K., Interpretation of the magnetotelluric impedance tensor: regional induction and local telluric distortion, J. Geophys., 62, 119-127, 1988.

Chave, A. D. and A. G. Jones, Electric and magnetic field galvanic distortion decomposition of BC87 data, J. Geomag. Geoelec., 49, 767-789, 1997.

Chave, A. D. and J. T. Smith, On electric and magnetic galvanic distortion tensor decompositions, J. Geophys. Res., 99, 4669-4682, 1994.

Groom, R. W. and K. Bahr, Corrections for near surface effects: decomposition of the magnetotelluric impedance tensor and scaling corrections for regional resistivities: a tutorial, Surv. Geophys., 13, 341-379, 1992.

Groom, R. W. and R. C. Bailey, Decomposition of the magnetotelluric impedance tensor in the presence of local three-dimensional galvanic distortion, J. Geophys. Res., 94, 1913-1925, 1989.

Smith, J. T., Estimating galvanic-distortion magnetic fields in magnetotellurics, Geophys. J. Int., 130, 65-72, 1997.

Weaver, J. T., A. K. Agarwal, and X. H. Pu, Three-dimensional finitedifference modelling of the magnetic field in geo-electromagnetic induction, in Three Dimensional Electromagnetics, edited by M. J. Oristaglio and B. R. Spies, Geophysical Developments Series Vol. 7, pp. 426-443, Society of Exploration Geophysicists, Tulsa OK, 1999.

White, S. N., A. D. Chave, and J. H. Filloux, A look at galvanic distortion in the Tasman Sea and the Juan de Fuca plate, J. Geomag. Geoelectr., 49, 1373-1386, 1997.

A. K. Agarwal (e-mail: numod@uvvm.uvic.ca) and J. T. Weaver (e-mail: weaver@phys.uvic.ca) 